\title{
Preparation and characteristics of nanotetrapods CdSe-polymer hybrid solar cells
}

\author{
QI PANG*, LI JUAN ZHAO ${ }^{\dagger}$, JUN HE ${ }^{\ddagger}$, CHUN JIE LIANG ${ }^{\ddagger}$, AI MIAO QIN" and \\ JIAN NONG WANG ${ }^{\#}$ \\ College of Chemistry and Chemical Engineering, Guangxi University, Nanning, Guangxi 530004, China \\ ${ }^{\dagger}$ Applied Physics Department, Donghua University, Shanghai 201620, China \\ ¥College of Chemistry and Material, Yulin Normal University, Yulin, Guangxi 537000, China \\ \#Physics Department, Hong Kong Uniersity of Science and Technology, Clear Water Bay, Hong Kong, China
}

MS received 29 July 2012; revised 27 October 2012

\begin{abstract}
Tetrapod-shaped CdSe nanocrystals were obtained using a simple method. HRTEM shows that the average size of the tetrapod core are about $4 \mathrm{~nm}$, widths of the tetrapod arms are about $4 \mathrm{~nm}$ and lengths of the arms are about $20 \mathrm{~nm}$. XRD patterns reveal that the OA-capped CdSe tetrapod nanocrystals have a hexagonal wurtzite structure. A hybrid solar cell fabricated based on an 8:1 (w/w) blend of CdSe tetrapod nanocrystals and MEH-PPV showed a maximum power conversion efficiency of $0.46 \%$ under an air mass 1.5 global condition. The effects of nanocrystal composition on the photovoltaic properties of hybrid solar cells based on nanotetrapods CdSe/MEHPPV were investigated. The power conversion efficiency values initially increased and then decreased, but the $V_{O C}$ values linearly decreased from 1.1 to $0.25 \mathrm{~V}$ with increased CdSe nanotetrapod in the blend film. The significant quenching of PL with increased nanotetrapod concentration indicated photo-induced charge transfer between MEH-PPV and CdSe.
\end{abstract}

Keywords. CdSe; nanotetrapods; conjugated polymer; hybrid solar cells.

\section{Introduction}

Studies on hybrid inorganic nanocrystal/polymer bulk heterojunction solar cells using high-electron-mobility nanocrystals that can overcome charge transport limitations as high-electron-affinity acceptors have recently increased (Saunders and Turner 2008; Gonzalez-Valls and Lira-Cantu 2009; Helgesen et al 2010). Nanocrystals of inorganic semiconductors such as CdSe (Dayal et al 2010), CdTe (Shiga et al 2006), $\mathrm{PbS}$ (Gunes et al 2005), $\mathrm{ZnO}$ (Oosterhout et al 2009), and $\mathrm{TiO}_{2}$ (Zeng et al 2009) also act as good electron acceptors from conjugated polymers and have been widely studied in relation to hybrid solar cell fabrication. However, efficiency of photovoltaic devices made with spherical nanocrystals is limited by the problem of electron extraction through the nanocrystal network (Greenham et al 1996; Ginger and Greenham 1999). The use of nanorods instead of spherical nanocrystals has been shown to result in significantly higher efficiencies because of the smaller number of interparticle hops necessary for electrons to leave the device. However, nanorods have a tendency to lie in the plane of the film, which is not the optimum arrangement

\footnotetext{
*Author for correspondence (pqigx@ 163.com)
}

for electron collection. In recent years, studies have shown that the performance of hybrid solar cells can be enhanced by incorporating $\mathrm{CdSe}$ tetrapods into the conjugated polymer poly[2-methoxy,5-(2'-ethylhexyloxy)-1,4phenylene-vinylene] (MEH-PPV) or poly(3-hexylthiophene) (P3HT) (Huynh et al 2002; Sun et al 2003, 2005; Lee et al 2011). Tetrapods, which have a natural branched structure, can be assembled into an ordered network structure that can maintain the characteristics of a vertically aligned nanorod array morphology. Consequently, electron transport and the performance of photovoltaic devices are improved. Nevertheless, the synthesis of branched nanostructures mostly involves trioctylphosphine oxide (TOPO) and alkylphosphonate acids, which are expensive, hazardous and toxic. By using a cadmium-oleic acid (OA) precursor, almost pure tetrapod-shaped CdSe capped with OA can be synthesized. OA is an environmentally friendly, cheaper and safer solvent compared with TOPO. Reports on the applications of OA-capped CdSe tetrapods blended with MEH-PPV in solar cells are limited. In the current study, OA-capped CdSe tetrapods with an arm length of about $20 \mathrm{~nm}$ were synthesized and photovoltaic devices based on blends of the CdSe nanotetrapods with the conjugated polymer MEH-PPV were fabricated. By investigating current-voltage $(I-V)$ efficiency characteristics, the effects of tetrapod composition on the performance of hybrid solar cells were discussed. 

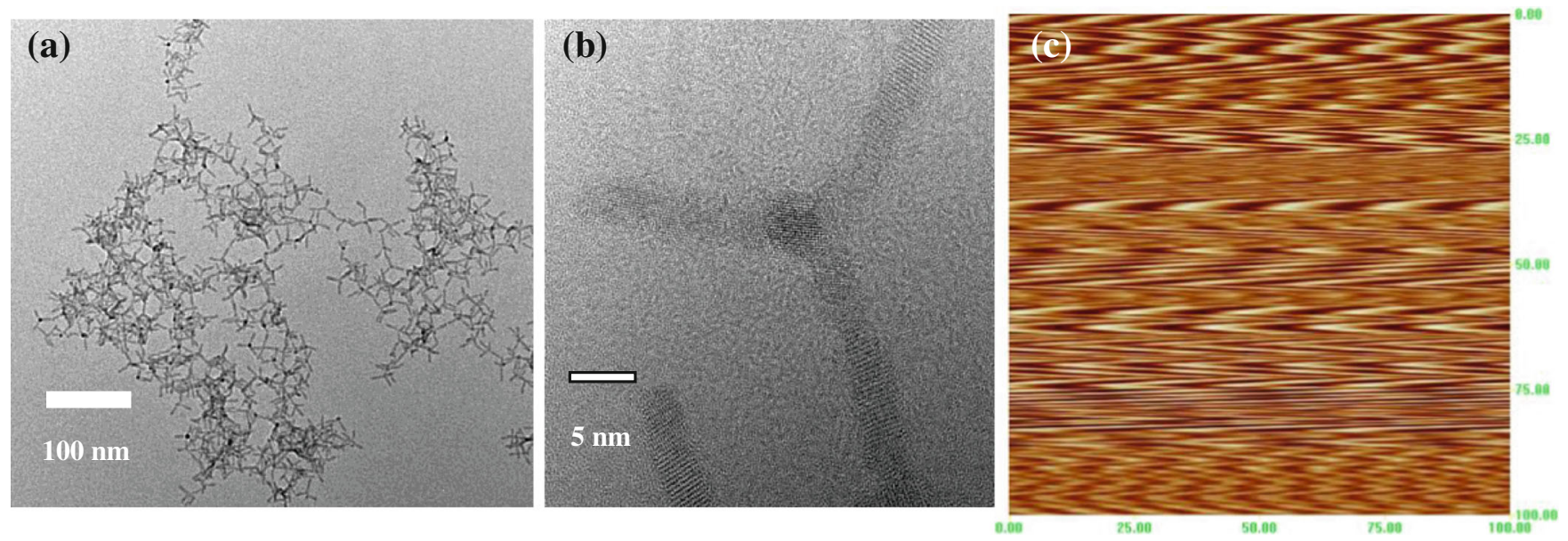

Figure 1. (a) Transmission electron microscopy images of CdSe nanocrystals, (b) high-resolution transmission electron microscopy images of CdSe nanocrystals and (c) atomic force microscopy topography images of CdSe tetrapod nanocrystal/MEH-PPV blend films (8:1, $\mathrm{w} / \mathrm{w})$. Scanned area is $100 \times 100 \mathrm{~nm}$ and vertical scale is $20 \mathrm{~nm}$.

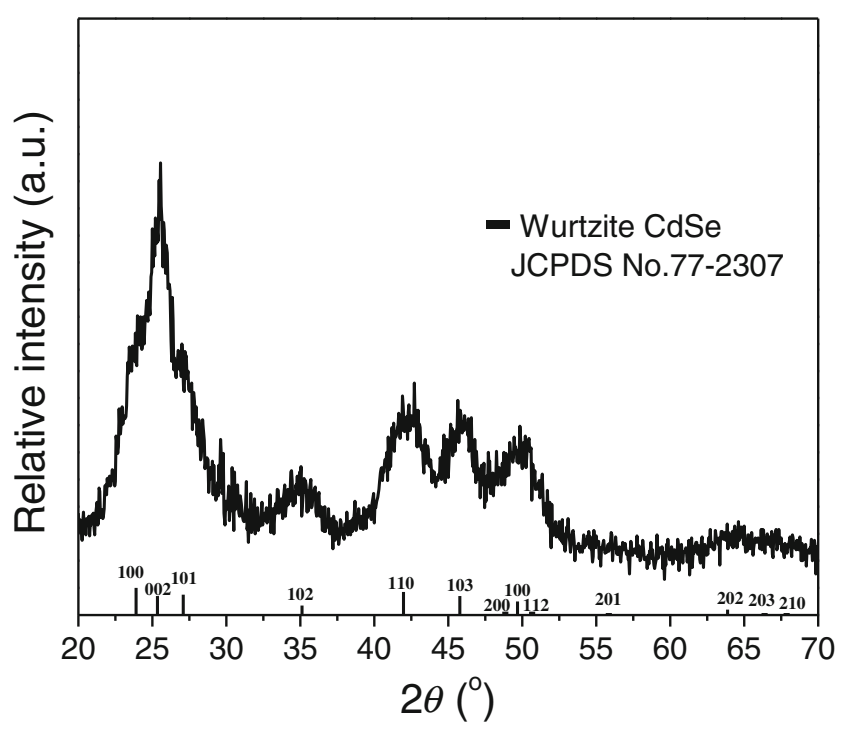

Figure 2. X-ray diffraction patterns of CdSe tetrapod nanocrystals. For comparison, reference peaks of bulk wurtzite CdSe (JCPDS no. 77-2307) are displayed.

\section{Experimental}

A mixture of $\mathrm{CdO}(5.4 \mathrm{mmol})$ and $\mathrm{OA}(90.0 \mathrm{mmol})$ was heated in a three-neck flask to $180{ }^{\circ} \mathrm{C}$ for $2 \mathrm{~h}$. Then, $9.6 \mathrm{~g}$ of a selenium-trioctylphosphine (TOP) solution (containing $0.96 \mathrm{~g}$ selenium) was quickly injected into this hot solution. The synthesis was carried out under $\mathrm{N}_{2}$ flow and the resulting solution was maintained at $180{ }^{\circ} \mathrm{C}$. After cooling to $50{ }^{\circ} \mathrm{C}, 10 \mathrm{~mL}$ of methanol and $5 \mathrm{~mL}$ of toluene were added to precipitate the nanoparticles, which were recovered by centrifugation. The precipitate was washed three times with methanol to remove excess ligands, and then dispersed into a mixture of chloroform/toluene $(90: 10, \mathrm{v} / \mathrm{v})$ at a concentration of $32 \mathrm{mg} / \mathrm{mL}$. Efficient photovoltaic devices were fabricated by spin casting a solution of $\mathrm{CdSe}$ tetrapods in MEH-PPV onto an indium tin oxide (ITO) glass substrate coated with poly(ethylene dioxythiophene) (PEDOT) doped with polystyrene sulfonic acid (PSS), a conducting polymer, with aluminum as the top contact.

The size, shape and crystal structures of the CdSe nanocrystals were examined using a JEOL 2010F highresolution transmission electron microscopy (HRTEM) system. The surface topography of the polymer/tetrapod films was studied by atomic force microscopy (HL-II). An X-ray diffraction (XRD) instrument (Rigaku/Dmax-IIIA; operated at $35 \mathrm{kV}$ and $25 \mathrm{~mA}$; $\mathrm{CuK} \alpha=1.5406 \AA$ ) was used for crystal phase identification. The power conversion efficiency (PCE) was calculated from the current density-voltage $(J-V)$ characteristics under an air mass 1.5 global (AM1.5G) solar simulated light irradiation of $100 \mathrm{~mW} / \mathrm{cm}^{2}$. The $J-V$ characteristics were measured by a semiconductor characterization system (Keithley 2400, Keithley Co., Ltd.) at room temperature. PL spectra were obtained using a standard set up with an $\operatorname{argon}$ laser $\left(\lambda_{\mathrm{ex}}=380 \mathrm{~nm}\right)$.

\section{Results and discussion}

Figure 1(a) is a low-magnification TEM image showing high yields of tetrapod-shaped CdSe nanocrystals. Based on a few such TEM images, the tetrapod yield is estimated to reach $80 \%$, which is much higher than those reported by other groups. HRTEM images (figure 1(b)) reveal that the average size of the tetrapod core is about $4 \mathrm{~nm}$, the widths of the tetrapod arms are about $4 \mathrm{~nm}$ and the lengths of the arms are about $20 \mathrm{~nm}$.

The surface topography of the polymer/tetrapod films was studied by atomic force microscopy, and the results are 
Table 1. Performance of PVC device based on CdSe/MEH-PPV with different weight ratios.

\begin{tabular}{lccccc}
\hline CdSe/MEH-PPV ratio (w/w) & $V_{\mathrm{oc}}(\mathrm{V})$ & $J_{\mathrm{sc}}(\mathrm{mA} / \mathrm{cm} 2)$ & FF $(\%)$ & ECE $(\%)$ & PS $(\mathrm{A} / \mathrm{W})$ \\
\hline $4: 1$ & $1 \cdot 10$ & 0.49 & 28.94 & 0.15 & $0 \cdot 01$ \\
$6: 1$ & 1.05 & 1.38 & 29.94 & 0.43 & 0.03 \\
$8: 1$ & 0.70 & $2 \cdot 04$ & 32.56 & 0.46 & $0 \cdot 04$ \\
$10: 1$ & 0.55 & 1.70 & 33.80 & 0.32 & 0.04 \\
$12: 1$ & 0.25 & 1.69 & 30.67 & 0.13 & $0 \cdot 05$ \\
\hline
\end{tabular}

\section{I-V of CdSe/MEHPPV with different ratio}

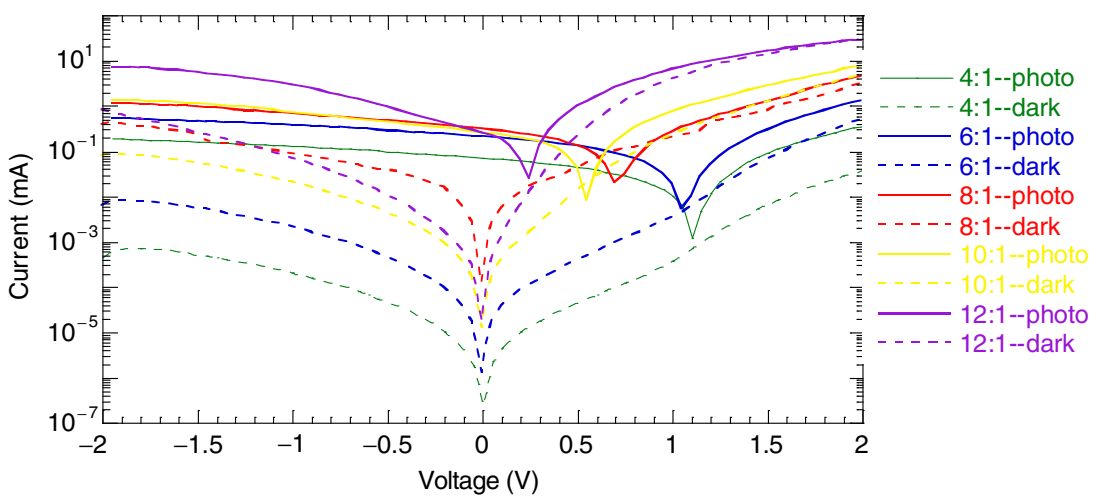

Figure 3. Effects of weight ratio of nanocrystals to polymer on photovoltaic properties.

shown in figure 1(c). The good quality of the interpenetrating network of the tetrapod and polymer can be reflected by morphology of the blend film. The uniformity of the film also indicates good dispersion of the CdSe tetrapods in $\mathrm{MEH}-$ PPV, which produces uniform surfaces. In hybrid solar cells, the transport of electrons and holes within an interpenetrating network to appropriate electrodes could be responsible for the changes in solar cell performance.

XRD pattern of CdSe tetrapod nanocrystals is shown in figure 2. A comparison of the XRD patterns of the asprepared nanocrystals and bulk CdSe crystals reveals that the OA-capped CdSe tetrapod nanocrystals have a hexagonal wurtzite structure.

Table 1 lists PCEs of the hybrid solar cells fabricated using the blend films with different weight ratios of $\mathrm{CdSe}$ nanotetrapods to MEH-PPV. With increased CdSe nanotetrapod content, PCE values initially increase and then decrease. A maximum PCE of $0.46 \%$, an open-circuit voltage $\left(V_{\mathrm{oc}}\right)$ of $0.7 \mathrm{~V}$, a short-circuit current density $\left(J_{\mathrm{sc}}\right)$ of $2.04 \mathrm{~mA} / \mathrm{cm}^{2}$ and a fill factor (FF) of 0.32 are obtained when the CdSe nanotetrapod content is $88.9 \mathrm{wt} \%(8: 1 \mathrm{w} / \mathrm{w})$ in the blend film (figure 3 and table 1). Generally, the maximum $V_{\mathrm{oc}}$ is determined by the difference between the work functions of the electrodes, PEDOT:PSS and aluminum, as well as between the lowest unoccupied energy level in the CdSe nanotetrapods and the highest occupied energy level in MEHPPV. However, figure 3 and table 1 show that the $V_{\text {oc }}$ values linearly decrease from 1.1 to $0.25 \mathrm{~V}$ with increased $\mathrm{CdSe}$ nanotetrapod content in the blend film. Further increased CdSe nanotetrapod content in the blend film possibly results in decreased polymer content, which may decrease the contribution of MEH-PPV to light absorption. Consequently, insufficiently generated photocarriers and charge distribution may result in reduced $V_{\text {OC }}$. On the other hand, increased $\mathrm{CdSe}$ nanotetrapod content in the blend film generates percolation pathways via both nanotetrapods and polymer components. As a result, the series $\left(R_{\mathrm{s}}\right)$ and shunt $\left(R_{\mathrm{sh}}\right)$ resistances decrease, which helps the transport of electrons and increases the photocurrent. At high concentrations (88.8 wt\% nanotetrapods), the nanotetrapods begin to form a connected network and PCE values increase. From the above results and analyses, the optimal blend ratio of CdSe nanotetrapods to MEH-PPV can be calculated as 8:1 (w/w).

Figure 4 shows PL efficiency of $\mathrm{CdSe}$ nanotetrapod/MEH-PPV composites as a function of nanotetrapod concentration by weight. From top to bottom, the curve is the PL spectra of blends with CdSe and MEH-PPV weight ratio of $0: 1,4: 1,6: 18: 1$ and 10:1 (w/w), respectively. When excited at $380 \mathrm{~nm}$, pure MEH-PPV shows PL peaks at around 500-750 nm. The main PL peak appears at $593 \mathrm{~nm}$ and one shoulder peak is centred at $637 \mathrm{~nm}$ due to the fine vibronic structure of MEH-PPV (the 0-0 and 0-1 emission bands, respectively). There is significant quenching of PL with increased nanotetrapod concentration. This result 


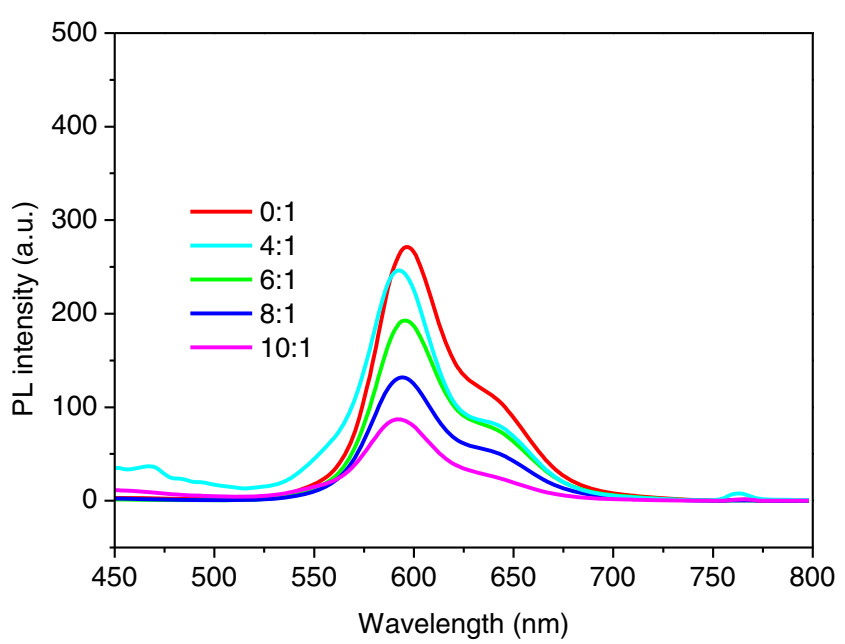

Figure 4. Photoluminescence efficiency of CdSe nanotetrapod/MEH-PPV composites as a function of nanotetrapod concentration. PL spectra from top to bottom are for $0: 1,4: 1,6: 1,8: 1$ and 10:1 (w/w) CdSe/MEH-PPV blends.

provides evidence for charge transfer because once the singlet exciton dissociates, it can no longer decay radioactively to the ground state. PL from MEH-PPV is quenched in the blend, directly indicating photoinduced charge transfer between MEH-PPV (as donor) and CdSe (as acceptor).

\section{Conclusions}

In conclusion, almost pure CdSe nanotetrapods capped with OA were obtained. The widths of the tetrapod arms are about $4 \mathrm{~nm}$ and the lengths are about $20 \mathrm{~nm}$. A hybrid solar cell fabricated based on the blend of CdSe tetrapod nanocrystals and MEH-PPV with a weight ratio of 8:1 shows a maximum power conversion efficiency of $0.46 \%$ under AM1.5G. With increased CdSe nanotetrapods in the blend film, the PCE values initially increase and then decrease, but the $V_{\text {oc }}$ values linearly decrease from 1.1 to $0.25 \mathrm{~V}$. Significant
PL quenching with increased nanotetrapods concentration indicates photo-induced charge transfer between MEH-PPV and CdSe.

\section{Acknowledgements}

This research was supported by the National Science Foundation of China (grant Nos. 2086300821363027), and the Natural Science Foundation of Guangxi Province (Nos. 2011GXNSFA018060) and the Project Sponsored by the Scientific Research Foundation of GuangXi University (Grant Nos. XGZ130765).

\section{References}

Dayal S, Kopidakis N, Olson D C, Ginley D S and Rumbles G 2010 Nano. Lett. 10239

Ginger D S and Greenham N C 1999 Phys. Rev. B59 10622

Gonzalez-Valls I and Lira-Cantu M 2009 Energy Environ. Sci. 219

Greenham N C, Peng X G and Alivisatos A P 1996 Phys. Rev. B54 17628

Gunes S, Fritz K P, Neugebauer H, Sariciftci N S, Kumar S and Scholes G D 2005 Sol. Energ. Mat. Sol. C. 91420

Helgesen M, Søndergaard R and Krebs F C 2010 J. Mater. Chem. 2036

Huynh W U, Dittmer J J and Alivisatos A P 2002 Science 2952425

Lee H, Kim S, Chung W S, Kim K and Kim D 2011 Sol. Energ. Mater. Sol. C. 95446

Oosterhout S D, Wienk M M, van Bavel S S, Thiedmann R, Koster L J, Gilot J, Loos J, Schmidt V and Janssen R A 2009 J. Nat. Mater. 8818

Saunders B R and Turner M L 2008 Adv. Colloid Interfac. Sci. 1381

Shiga T, Takechi K and Motohiro T 2006 Sol. Energ. Mater. Sol. C. 901849

Sun B Q, Marx E and Greenham N C 2003 Nano. Lett. 3961

Sun B Q, Snaith H J, Dhoot A S, Westenhoff S and Greenham N C 2005 J. Appl. Phys. 97014914

Zeng T W, Lo H H, Chang C H, Lin Y Y, Chen C W and Su W F 2009 Sol. Energ. Mat. Sol. C. 93952 